\title{
Universiteit
}

Leiden

The Netherlands

\section{Quantum critical behaviour in a high-T-c superconductor}

Marel, D. van der; Molegraaf, H.J.A.; Zaanen, J.; Nussinov, Z.; Carbone, F.; Damascelli, A.; ... ; Li, M.

\section{Citation}

Marel, D. van der, Molegraaf, H. J. A., Zaanen, J., Nussinov, Z., Carbone, F., Damascelli, A., ... Li, M. (2003). Quantum critical behaviour in a high-T-c superconductor. Nature, 425, 271-274. Retrieved from https://hdl.handle.net/1887/5138

Version: $\quad$ Not Applicable (or Unknown)

License:

Downloaded from: https://hdl.handle.net/1887/5138

Note: To cite this publication please use the final published version (if applicable). 
10. Jones, B., Ghose, S., Clemens, J. P., Rice, P. R. \& Pedrotti, L. M. Photon statistics of a single atom laser. Phys. Rev. A 60, 3267-3275 (1999).

11. Kilin, S. Ya. \& Karlovich, T. B. Single-atom laser: Coherent and nonclassical effects in the regime of a strong atom-field correlation. JETP 95, 805-819 (2002).

12. McKeever, J. et al. State-insensitive cooling and trapping of single atoms in an optical cavity. Phys. Rev Lett. 90, 133602 (2003)

13. Sargent, M. III, Scully, M. O. \& Lamb, W. E. Jr Laser Physics (Addison-Wesley, Reading, MA, 1974).

14. Haken, H. Laser Theory (Springer, Berlin, 1984)

15. Mandel, L. \& Wolf, E. Optical Coherence and Quantum Optics (Cambridge Univ. Press, Cambridge, 1995)

16. Carmichael, H. J. Statistical Methods in Quantum Optics 1 (Springer, Berlin, 1999).

17. Gardiner, C. W. \& Zoller, P. Quantum Noise (Springer, Berlin, 2000)

18. Kimble, H. J. Strong interactions of single atoms and photons in cavity QED. Phys. Scr. T76, 127-137 (1998).

19. Raithel, G., Wagner, C., Walther, H., Narducci, L. M. \& Scully, M. O. Cavity Quantum Electrodynamics (ed. Berman, P.) 57-121 (Academic, San Diego, 1994).

20. Haroche, S. \& Raimond, J. M. Cavity Quantum Electrodynamics (ed. Berman, P.) 123-170 (Academic, San Diego, 1994).

21. Meystre, P. in Progress in Optics Vol. XXX (ed. Wolf, E.) 261-355 (Elsevier, Amsterdam, 1992).

22. An, K. \& Feld, M. S. Semiclassical four-level single-atom laser. Phys. Rev. A 56, 1662-1665 (1997).

23. Chang, R. K. \& Campillo, A. J. (eds) Optical Processes in Microcavities (World Scientific, Singapore, 1996)

24. Vahala, K. J. Optical microcavities. Nature 424, 839-846 (2003).

25. Ye, J., Vernooy, D. W. \& Kimble, H. J. Trapping of single atoms in cavity QED. Phys. Rev. Lett. 83, 4987-4990 (1999).

26. Rice, P. R. \& Carmichael, H. J. Photon statistics of a cavity-QED laser: A comment on the laser-phasetransition analogy. Phys. Rev. A 50, 4318-4329 (1994).

27. Boozer, A. D., Boca, A., Buck, J. R., McKeever, J. \& Kimble, H. J. Comparison of theory and experiment for a one-atom laser in a regime of strong coupling. Phys. Rev. A (submitted); preprint available at 〈http://lanl.arxiv.org/archive/quant-ph〉.

28. Metcalf, H. J. \& van der Straten, P. Laser Cooling and Trapping (Springer, New York, 1999).

29. Boiron, D. et al. Laser cooling of cesium atoms in gray optical molasses down to $1.1 \mu \mathrm{K}$. Phys. Rev. A 53, R3734-R3737 (1996).

30. Corwin, K. L., Kuppens, S. J. M., Cho, D. \& Wieman, C. E. Spin-polarized atoms in a circularly polarized optical dipole trap. Phys. Rev. Lett. 83, 1311-1314 (1999).

Supplementary Information accompanies the paper on www.nature.com/nature.

Acknowledgements We gratefully acknowledge interactions with K. Birnbaum, C.-W. Chou, A. C. Doherty, L.-M. Duan, T. Lynn, T. Northup, S. Polyakov and D. M. Stamper-Kurn. This work was supported by the National Science Foundation, by the Caltech MURI Center for Quantum Networks, and by the Office of Naval Research.

Competing interests statement The authors declare that they have no competing financia interests.

Correspondence and requests for materials should be addressed to H.J.K (hjkimble@caltech.edu).

\section{Quantum critical behaviour in a high- $T_{c}$ superconductor}

D. van der Marel ${ }^{1 \star}$, H. J. A. Molegraaf ${ }^{1 \star}$, J. Zaanen ${ }^{2}$, Z. Nussinov ${ }^{2 \star}$, F. Carbone ${ }^{1 \star}$, A. Damascelli ${ }^{3 \star}$, H. Eisaki ${ }^{3 \star}$, M. Greven ${ }^{3}$, P. H. Kes ${ }^{2} \&$ M. Li

${ }^{1}$ Materials Science Centre, University of Groningen, 9747 AG Groningen,

The Netherlands

${ }^{2}$ Leiden Institute of Physics, Leiden University, 2300 RA Leiden, The Netherlands ${ }^{3}$ Department of Applied Physics and Stanford Synchrotron Radiation Laboratory, Stanford University, California 94305, USA

* Present addresses: Département de Physique de la Matière Condensée, Université de Genève, $\mathrm{CH}-121$ Genève 4, Switzerland (D.v.d.M., H.J.A.M., F.C.); Los Alamos National Laboratories, Los Alamos, New Mexico 87545, USA (Z.N.); Department of Physics and Astronomy, University of British Columbia, Vancouver, British Columbia, V6T 1Z1, Canada (A.D.); Low-Temperature Physics Group, National Institute of Advanced Industrial Science and Technology, Umezono, Tsukuba, 305-8568, Japan (H.E.)

Quantum criticality is associated with a system composed of a nearly infinite number of interacting quantum degrees of freedom at zero temperature, and it implies that the system looks on average the same regardless of the time- and length scale on which it is observed. Electrons on the atomic scale do not exhibit such symmetry, which can only be generated as a collective phenomenon through the interactions between a large number of electrons. In materials with strong electron correlations a quantum phase transition at zero temperature can occur, and a quantum critical state has been predicted ${ }^{1,2}$, which manifests itself through universal power-law behaviours of the response functions. Candidates have been found both in heavy-fermion systems $s^{3}$ and in the high-transition temperature (high- $T_{\mathrm{c}}$ ) copper oxide superconductors ${ }^{4}$, but the reality and the physical nature of such a phase transition are still debated ${ }^{5-7}$. Here we report a universal behaviour that is characteristic of the quantum critical region. We demonstrate that the experimentally measured phase angle agrees precisely with the exponent of the optical conductivity. This points towards a quantum phase transition of an unconventional kind in the high- $T_{c}$ superconductors.

In the quantum theory of collective fields one anticipates order at small coupling constant, and for increasing coupling one expects at some point a phase transition to a quantum-disordered state. Quantum criticality in the copper oxides, if it exists, occurs as a function of charge carrier doping $x$, at a particular doping level $x_{\mathrm{c}}$ close to where the superconducting phase transition temperature reaches its maximum value. When this phase transition is continuous, a critical state is realized right at the transition, which is characterized by scale invariance resulting in the above-mentioned power-law response up to some (non-universal) high-energy cutoff $\Omega$.

The optical conductivity, $\sigma(\omega)=\sigma_{1}(\omega)+i \sigma_{2}(\omega)$, is the absorptive $\left(\sigma_{1}\right)$ and reactive $\left(\sigma_{2}\right)$ current response to a time-varying external electrical field of frequency $\omega$, and is usually expressed as the correlation function of the currents $\mathbf{j}\left(\tau_{1}\right)$ and $\mathbf{j}\left(\tau_{2}\right)$ at times $\tau_{1}$ and $\tau_{2}$, which is $\chi_{i j}\left(\tau_{1}, \tau_{2}\right)=\left\langle\mathbf{j}\left(\tau_{1}\right), \mathbf{j}\left(\tau_{2}\right)\right\rangle$, by the Kubo formula. In Fig. 1 we present the experimental optical conductivity function $\sigma_{1}(\omega)$ of an optimally doped $\mathrm{Bi}_{2} \mathrm{Sr}_{2} \mathrm{Ca}_{0.92} \mathrm{Y}_{0.08} \mathrm{Cu}_{2} \mathrm{O}_{8+\delta}$ single crystal

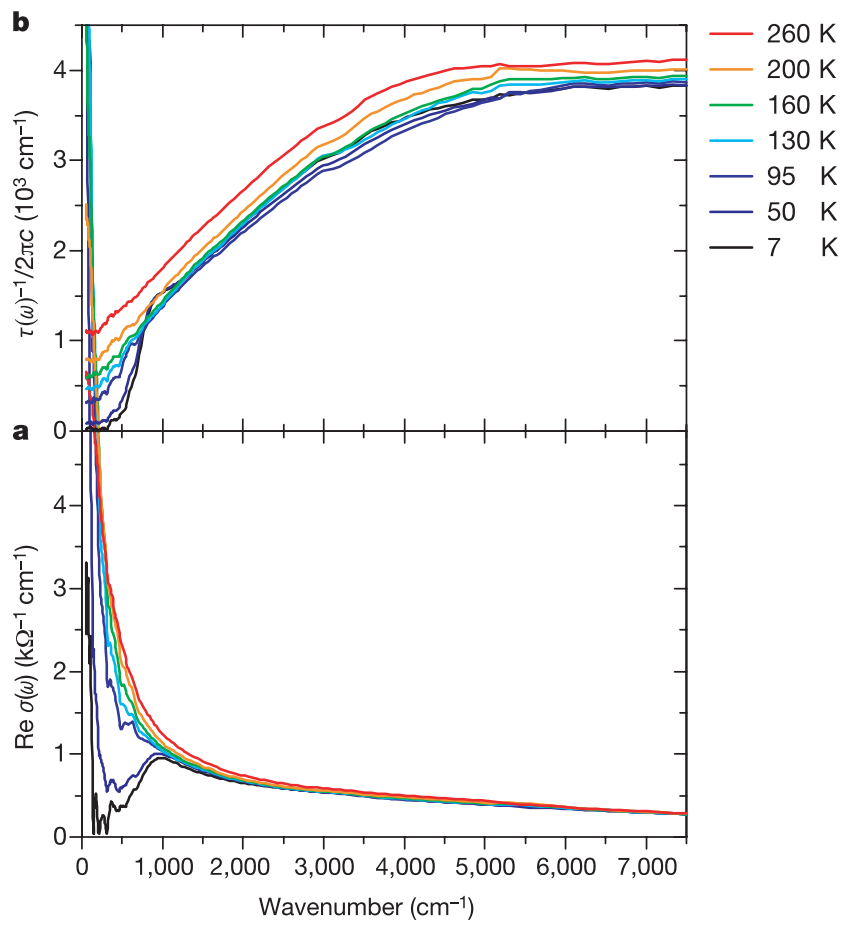

Figure 1 Optical properties along the copper-oxygen planes of $\mathrm{Bi}_{2} \mathrm{Sr}_{2} \mathrm{Ca}_{0.92} \mathrm{Y}_{0.08} \mathrm{Cu}_{2} \mathrm{O}_{8+\delta}$ for a selected number of temperatures. $\mathbf{a}$, Optical conductivity and $\mathbf{b}$, the frequency dependent scattering rate defined as $1 / \tau(\omega)=\operatorname{Re}\left\{\omega_{\mathrm{p}}^{2} / 4 \pi \sigma(\omega)\right\}$ (see Methods). The relatively high transition temperature $\left(T_{\mathrm{C}}=96 \mathrm{~K}\right)$ of this crystal compared to previous reports on Bi-2212 is caused by the partial substitution of yttrium on the calcium sites. 
( $T_{\mathrm{c}}=96 \mathrm{~K}$; H.E. et al., manuscript in preparation). In order to facilitate comparison with earlier publications ${ }^{8-10}$ we also present $1 /$ $\tau(\omega)$ for a number of temperatures, adopting $\omega_{\mathrm{p}} / 2 \pi c=$ $19,364 \mathrm{~cm}^{-1}$ for the plasma frequency (where $c$ is the velocity of light). The scattering rate $1 / \tau(\omega)$ increases approximately linearly as a function of frequency, and when the temperature $T$ is increased, the $1 / \tau(\omega)$ curves are shifted vertically proportional to $T$. The notion that $1 / \tau(\omega, T) \sim \omega+T$ in the copper oxides forms one of the centre pieces of the marginal Fermi liquid model ${ }^{1,11}$, and it has been shown to be approximately correct in a large number of experimental papers $^{8-10}$. This phenomenology stresses the importance of temperature as the (only) relevant energy scale near optimal doping, which has motivated the idea that optimally doped copper oxides are close to a quantum critical point ${ }^{1}$. As can be seen in Fig. $1,1 / \tau(\omega)$ has a negative curvature in the entire infrared region for all temperatures, and it saturates at around $5,000 \mathrm{~cm}^{-1}$. Although this departure from linearity may seem to be a minor detail, we will see that it is a direct consequence of the quantum critical scaling of the optical conductivity.

If a quantum phase transition indeed occurs at optimal doping $x=x_{\mathrm{c}}$, then three major frequency regimes of qualitatively different behaviour are expected ${ }^{2}$ : (1) $\omega<T$; (2) $T<\omega<\Omega$; (3) $\Omega<\omega$. As we now report, we find direct indications of these regimes in our optical conductivity data.

Region $1(\omega<T)$ corresponds to measurement times long compared to the compactification radius of the imaginary time, $L_{\mathrm{T}}=\hbar / k_{\mathrm{B}} T$ (see Methods). Some ramifications have already been discussed above. In addition, Sachdev ${ }^{2}$ showed that in this regime the system exhibits a classical relaxational dynamics characterized by a relaxation time $\tau_{\mathrm{r}}=A L_{\mathrm{T}}$ ( $A$ is a numerical prefactor of order 1$)$, reflecting that temperature is the only scale in the system. For the low frequency regime we expect a Drude form $\sigma_{1}(\omega)=$ $(4 \pi)^{-1} \omega_{\mathrm{pr}}^{2} \tau_{\mathrm{r}} /\left(1+\omega^{2} \tau_{\mathrm{r}}^{2}\right)$, where $\omega_{\mathrm{pr}}$ is the plasma frequency. Then $T \sigma_{1}(\omega, T)$ becomes a universal function of $\omega / T$, at least up to a number of order one:

$$
\frac{\hbar}{k_{B} T \sigma_{1}(\omega, T)}=\frac{4 \pi}{A \omega_{p r}^{2}}\left(1+A^{2}\left(\frac{\hbar \omega}{k_{B} T}\right)^{2}\right)
$$

In the inset of Fig. 2 we display $\hbar /\left(k_{\mathrm{B}} T \sigma_{1}\right)$ as a function of $u=$

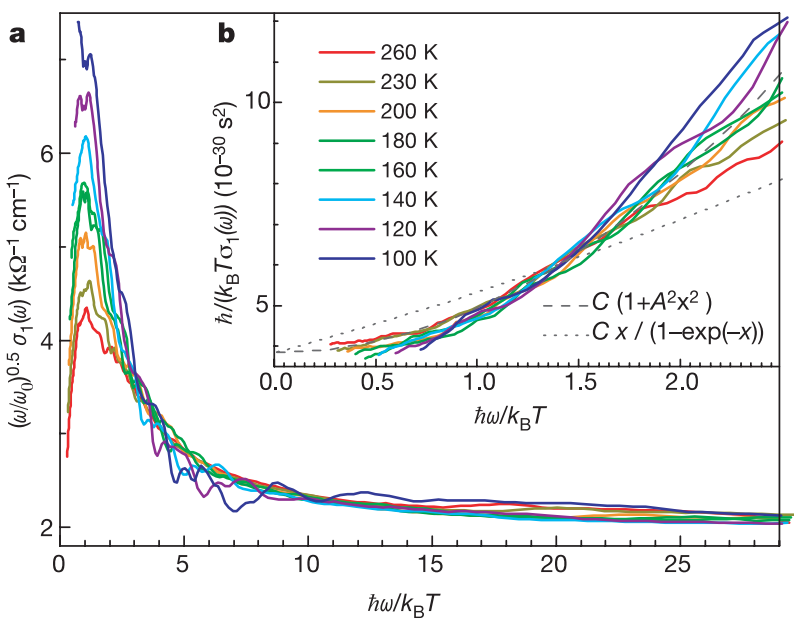

Figure 2 Temperature/frequency scaling behaviour of the real part of the optical conductivity of $\mathrm{Bi}_{2} \mathrm{Sr}_{2} \mathrm{Ca}_{0.92} \mathrm{Y}_{0.08} \mathrm{Cu}_{2} \mathrm{O}_{8+\delta}$. The sample is the same as in Fig. 1. In a, the data are plotted as $\left.\left(\omega / \omega_{0}\right)^{0.5} \sigma_{1}(\omega, T)\right)$. The collapse of all curves on a single curve for $\hbar \omega / k_{\mathrm{B}} T>3$ demonstrates that in this $\omega / T$-region the conductivity obeys $\sigma_{1}(\omega, T)=$ $\omega^{-0.5} \mathrm{~g}(\omega / T)=T^{-0.5} h(\omega / T)$. Note that $g(u)=u^{0.5} h(u)$. In $\mathbf{b}$, the data are presented as $\hbar /\left(k_{\mathrm{B}} T \sigma_{1}(\omega, T)\right)$, demonstrating that for $\hbar \omega / k_{\mathrm{B}} T<1.5$ the conductivity obeys $\sigma_{1}(\omega, T)=T^{-1} f(\omega / T)$. $\hbar \omega / k_{\mathrm{B}} T$. Clearly the data follow the expected universal behaviour for $u<1.5$, with $A=0.77$. The experimental data are in this regard astonishingly consistent with Sachdev's predictions, including $A \approx 1$. From the fitted prefactor we obtain $\omega_{\mathrm{pr}} /$ $2 \pi c=9,597 \mathrm{~cm}^{-1}$. Above we have already determined the total spectral weight of the free carrier response, $\left(\omega_{\mathrm{p}}\right)$ $2 \pi c)^{2}=19,364^{2} \mathrm{~cm}^{-2}$. Hence the classical relaxational response contributes $25 \%$ of the free carrier spectral weight. These numbers agree with the results and analysis of Quijada et al. ${ }^{8}$. This spectral weight collapses into the condensate peak at $\omega=0$ when the material becomes superconducting ${ }^{8}$. In Fig. 2 we also display the scaling function proposed by Prelovsek ${ }^{12}, \sigma_{1}(\omega)=C(1-$ $\left.\exp \left(-\hbar \omega / k_{\mathrm{B}} T\right)\right) / \omega$. The linear frequency dependence of this formula for $\hbar \omega / k_{\mathrm{B}} T \ll 1$ is clearly absent from the experimental data. The universal dependence of $T \sigma_{1}(\omega, T)$ on $\omega / T$ also contradicts the "cold spot model"13, where $T \sigma_{1}(\omega, T)$ has a universal dependence on $\omega / T^{2}$.

In region $2(T<\omega<\Omega)$ we can probe directly the scale invariance of the quantum critical state. Let us now introduce the scaling relation along the time axis, as follows from elementary consideration. The euclidean (that is, imaginary time) correlator has to be known in minute detail in order to enable the analytical continuation to real (experimental) time. However, in the critical state invariance under scale transformations fixes the functional form of the correlation function completely: It has to be an algebraic function of imaginary time. Hence, it is also an algebraic function of Matsubara frequency $\omega_{\mathrm{n}}=2 \pi n / L_{T}$, and the analytical continuation is unproblematic: (1) Scale invariance implies that $\sigma_{1}(\omega)$ and $\sigma_{2}(\omega)$ have to be algebraic functions of $\omega$, (2) causality forces the exponent to be the same for $\sigma_{1}(\omega)$ and $\sigma_{2}(\omega)$, and (3) time reversal
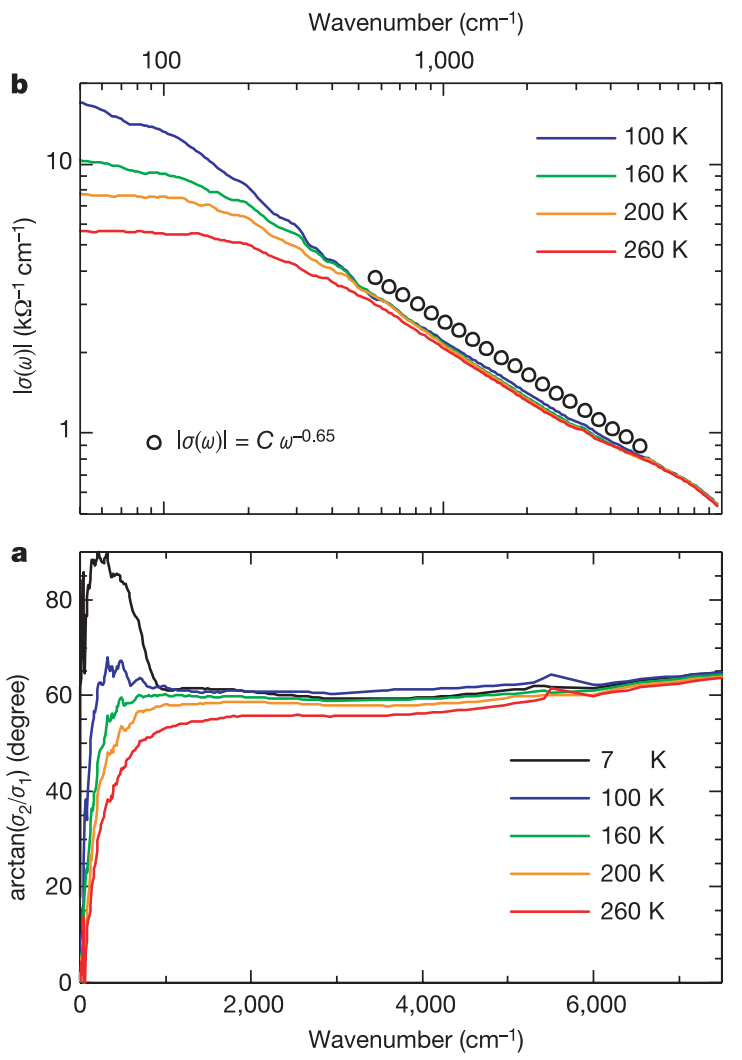

Figure 3 Universal power law of the optical conductivity and the phase angle spectra of optimally doped $\mathrm{Bi}_{2} \mathrm{Sr}_{2} \mathrm{Ca}_{0.92} \mathrm{Y}_{0.08} \mathrm{Cu}_{2} \mathrm{O}_{8+\delta}$. The sample is the same as in Fig. 1. In a, the phase function of the optical conductivity, $\operatorname{Arg}(\sigma(\omega))$ is presented. In $\mathbf{b}$, the absolute value of the optical conductivity is plotted on a double logarithmic scale. The open symbols correspond to the power law $|\sigma(\omega)|=C \omega^{-0.65}$. 
symmetry, implying $\sigma(\omega)=\sigma^{*}(-\omega)$, fixes the absolute phase of $\sigma(\omega)$. Taken together

$$
\sigma(\omega)=C(-i \omega)^{\gamma-2}=C \omega^{\gamma-2} e^{i \pi(1-\gamma / 2)}
$$

Hence the phase angle relating the reactive and absorptive parts of the conductivity, $\arctan \left(\sigma_{2} / \sigma_{1}\right)=(2-\gamma) \cdot 90^{\circ}$, is frequency independent and should be set by the critical exponent $\gamma$. Power-law behaviour of the optical conductivity of the copper oxides has been reported previously ${ }^{14,15}$, but to our knowledge the relation between the phase and the exponent has not been addressed in the literature. In Fig. 3 we display the frequency dependence of $|\sigma|$ in a log-log plot, and the phase in a linear plot. Although the temperature dependence 'leaks out' to surprisingly high frequency in the latter, the data are remarkably consistent with equation (2) for $\omega$ between $k_{\mathrm{B}} T$ and $7,500 \mathrm{~cm}^{-1}$. The observed power law of the conductivity, $|\sigma|=C / \omega^{0.65}$ corresponds to $\gamma=1.35$, and the value of the phase, $\arctan \left(\sigma_{2} / \sigma_{1}\right)=60^{\circ} \pm 2^{\circ}$, implies that $\gamma=1.33 \pm 0.04$. The good consistency of $\gamma$ obtained from two experimental quantities (that is, the exponent of a power law and the phase) is a strong test of the validity of equation (2). Frequency independence of the phase in region 2 and agreement between the two power laws (one from $\sigma(\omega)$ and the other from the phase angle spectrum) are unique properties of slightly overdoped samples, as demonstrated by Fig. 4, where we present the phase function for optimally $\operatorname{doped}^{16}\left(T_{\mathrm{c}}=88 \mathrm{~K}\right)$, underdoped ${ }^{16}\left(T_{\mathrm{c}}=66 \mathrm{~K}\right)$, and overdoped $\left(T_{\mathrm{c}}=77 \mathrm{~K}\right)$ single crystals of $\mathrm{Bi}_{2.23} \mathrm{Sr}_{1.9} \mathrm{Ca}_{0.96} \mathrm{Cu}_{2} \mathrm{O}_{8+\delta}$ with different oxygen concentrations ${ }^{17}$. The observed trend for different dopings suggests that optimal doping, with $T_{\mathrm{c}}=88 \mathrm{~K}$, and overdoping, with $T_{\mathrm{c}}=77 \mathrm{~K}$, are lower and upper carrier concentrations where the optical conductivity obeys equation (2).

Because the phase is constant, the frequency dependent scattering rate $1 / \tau(\omega)=\operatorname{Re}\left\{\omega_{\mathrm{p}}^{2} / 4 \pi \sigma(\omega)\right\}=C \omega^{2-\gamma}=C \omega^{0.65}$ can not be a linear function of frequency (but note that $1 / \tau^{\star}(\omega)$ is linear ${ }^{18}$, see Methods). Our findings disqualify directly theories that do not incorporate a manifest temporal scale invariance. Luttinger liquids are quantum critical states of matter, and Anderson's results ${ }^{18}$ based on onedimensional physics are therefore of the correct form, equation (2). The exponent $\gamma=4 / 3$ is within the range considered by Anderson, but differs significantly from the prediction based on the "cold spot" model $^{13}$, providing $\sigma(\omega) \sim(-i \omega)^{-0.5}$, which corresponds to $\gamma=3 / 2$.

Let us now turn to the temperature dependence of the optical conductivity. From Fig. 2 we see that in this region the conductivity crosses over to a different dependence on $\omega$ and $T$ : for $\hbar \omega / k_{\mathrm{B}} T>3$ the experimental data are seen to collapse onto a curve of the form $\sigma_{1}(\omega, T)=T^{-0.5} h(\omega / T)$, where $h(u)$ is a universal function. For $u>3, h(u)$ has a weak power-law dependence corresponding to a frequency dependence $\sigma_{1}(\omega, T) \sim \omega^{-0.65}$. According to the simplest scaling hypothesis, $\sigma_{1}(\omega, T) \sim T^{-\mu} h^{\prime}(\omega / T)$ with $h^{\prime}(u) \rightarrow$ constant and $h^{\prime}(u) \rightarrow u^{-\mu}$ in the limits $u \rightarrow 0$ and $u \gg 1$, respectively. Although this energy-temperature scaling is roughly satisfied in the high frequency regime, it is strongly violated at low frequencies, because the success of equation (1) in the regime for $\hbar \omega / k_{\mathrm{B}} T<1.5$ (see Fig. 2) implies an exponent $\mu=1$ at low frequencies instead. Bernhoeft has noticed a similar problem in the context of the heavyfermion critical points ${ }^{19}$.

Region $3(\omega>\Omega)$ necessarily has a different behaviour of the optical conductivity, based on the following simple argument: the spectral weight of the optical conductivity integrated over all frequencies is set by the f-sum rule. However, since $\gamma>1$, the integration over all frequencies of $\operatorname{Re} \sigma(\omega)$ of the form of equation (2) diverges. Hence we expect a crossover from the constant phase angle $\operatorname{Arg} \sigma(\omega)=(2-\gamma) \cdot 90^{\circ}$ to the asymptotic value $\operatorname{Arg} \sigma(\infty)=90^{\circ}$. The details of the frequency dependence of $\sigma(\omega)$ at the crossover point $\Omega$ depend on the microscopic details of the system. A (non-universal) example of an ultraviolet regularization with the required properties is ${ }^{20} \sigma(\omega)=\left(n e^{2} / m\right)(-i \omega)^{\gamma-2} \times$ $(\Omega-i \omega)^{1-\gamma}$. Indeed the phase functions show a gradual upward departure from the plateau value for frequencies exceeding $5,000 \mathrm{~cm}^{-1}$ (Fig. 3). This indicates that the 'ultraviolet' cut-off is on the order of $1 \mathrm{eV}$.

Do our observations shed light on the enigmatic origin of the quantum criticality? In fact, they point unambiguously at three surprising features. First, the current correlator behaves singularly, and this implies that the electromagnetic currents themselves are the order parameter fields responsible for the criticality. Second, the criticality persists up to surprisingly high energies. Third, we have seen that the optical conductivity curves collapse on $\sigma_{1}(\omega, T)=$ $T^{\mu} h(\omega / T)$, where $\mu=1$ for $\omega / T<1.5$, while $\mu \sim 0.5$ for $\omega / T>3$. This disqualifies many theoretical proposals. Much of the intuition regarding quantum criticality is based on the rather well understood quantum phase transitions in systems composed of bosons. A canonical example is the insulator-superconductor transition in two space dimensions ${ }^{21}$ where the optical conductivity is found to precisely obey the energy-temperature scaling hypothesis ${ }^{22}$,

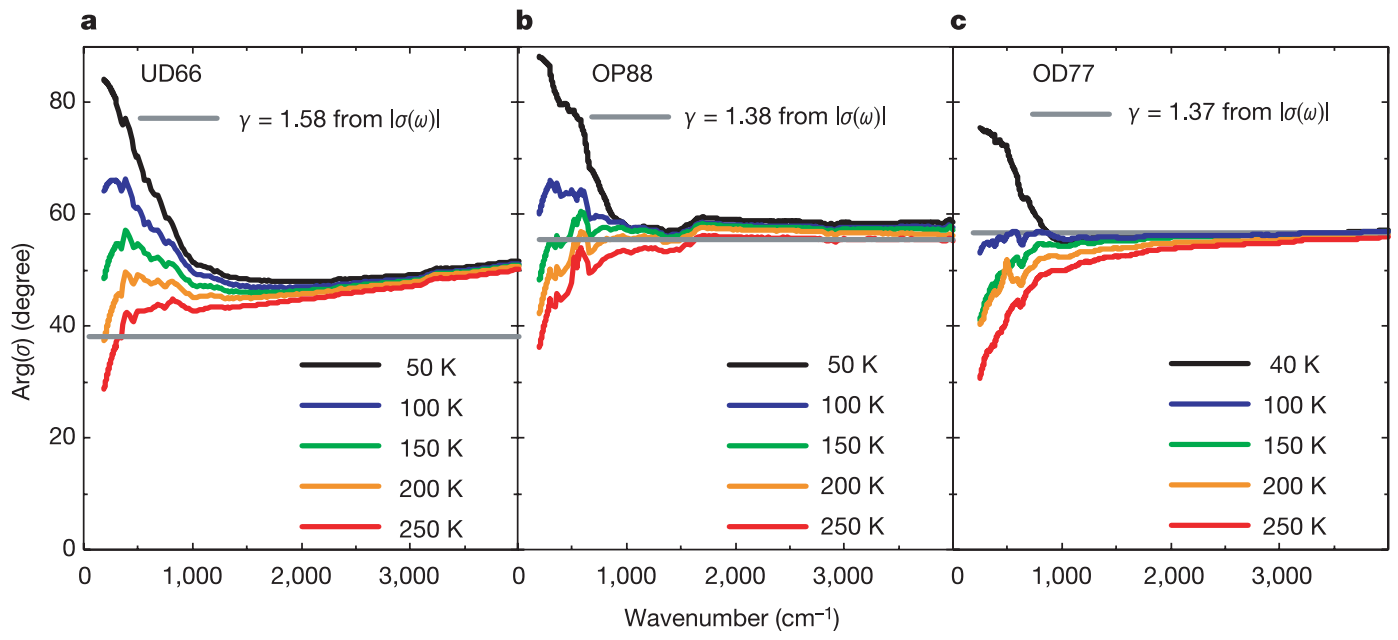

Figure 4 Phase of $\sigma(\omega)$ of $\mathrm{Bi}_{2.23} \mathrm{Sr}_{1.9} \mathrm{Ca}_{0.96} \mathrm{Cu}_{2} \mathrm{O}_{8+\delta}$ at various doping levels. a, Underdoped $\left(T_{\mathrm{C}}=66 \mathrm{~K}\right)$; $\mathbf{b}$, optimally doped $\left(T_{\mathrm{C}}=88 \mathrm{~K}\right)$; and $\mathbf{c}$, overdoped $\left(T_{\mathrm{c}}=77 \mathrm{~K}\right)$. Solid lines: exponent from $|\sigma(\omega)|$ between 1,000 and $5,000 \mathrm{~cm}^{-1}$. These crystals were grown at relatively low partial pressures ( $25 \mathrm{mbar}$ ) of oxygen, resulting in high-quality underdoped crystals with $T_{\mathrm{c}}$ as low as $65 \mathrm{~K}$, and with sharp superconducting transitions $\delta T_{\mathrm{c}} \leq 2.5 \mathrm{~K}$. The optimally doped and overdoped crystals were obtained by post-annealing in oxygen. At optimal doping, $T_{\mathrm{c}}$ turns out to be somewhat lower than the highest values reported in $\mathrm{Bi}-2212$ owing to a slightly different cation ratio. 
characterized by a single exponent $\mu=0$ governing both the frequency and temperature dependences ${ }^{2,22,23}$. Bosonic theory can be therefore of relevance in electron systems, but it requires the fermionic degrees of freedom to be bound in collective bosonic degrees of freedom at low energy.

In the copper oxides, it appears that the quantum criticality has to do with the restoration of the Fermi-liquid state in the overdoped regime characterized by a large Fermi surface. This implies that fermionic fluctuations play a central role in the quantum critical state, and their role has not yet been clarified theoretically. The absence of a single master curve for all values of $\omega / T$ is at variance with notions of quantum critical behaviour, and its understanding may require concepts beyond the standard model of quantum criticality. We close with the speculation that the presence of bosonic fluctuations and fermionic fluctuations in the copper oxides is pivotal in understanding the quantum critical behaviour near optimal doping of the copper oxides.

\section{Methods}

\section{Kubo formula}

The Kubo formalism establishes the relation between optical conductivity and currentcurrent correlation function:

$$
\sigma\left(i \omega_{n}, T\right)=\frac{N e^{2}}{m \omega_{n}}+\frac{1}{\omega_{n}} \int_{0}^{\mathrm{L}_{\mathrm{T}}} d \tau e^{i \omega_{n} \tau}\langle\mathbf{j}(\tau) \mathbf{j}(0)\rangle
$$

The first term, corresponding to perfect conductivity, is only relevant in the superconducting state, $\mathbf{j}(\tau)$ is the current operator at (imaginary) time $\tau$, while in the path integral formalism $L_{T}=\hbar / k_{\mathrm{B}} T$ is the compactification radius of the imaginary time. The angle brackets mean a trace over the thermal distribution at finite temperatures. The integration is over a finite segment of imaginary time, resulting in the optical conductivity at the Matsubara frequencies $i \omega_{\mathrm{n}}=2 \pi i n / L_{T}$ along the imaginar $y$ axis of the complex frequency plane. The conductivity at real frequency $\omega$ follows from analytical continuation.

\section{Experimental determination of the optical conductivity}

The most direct experimental technique, which provides the optical conductivity and its phase, is spectroscopic ellipsometry. Another popular approach is the measurement of the reflectivity amplitude over a wide frequency region. Kramers-Kronig relations then provide the phase of the reflectivity at each frequency, from which (with the help of Fresnel equations) the real and imaginary part of the dielectric function, $\epsilon(\omega)$, is calculated. We used reflectivity for $50 \mathrm{~cm}^{-1}<\omega / 2 \pi c<6,000 \mathrm{~cm}^{-1}$, and ellipsometry for $1,500 \mathrm{~cm}^{-1}<\omega / 2 \pi c<36,000 \mathrm{~cm}^{-1}$. This combination allows a very accurate determination of $\epsilon(\omega)$ in the entire frequency range of the reflectivity and ellipsometry spectra. Owing to the off-normal angle of incidence used with ellipsometry, the $a b$-plane pseudo-dielectric function had to be corrected for the $c$-axis admixture. We used previously published ${ }^{24} c$-axis optical constants of the same compound. The data files were generously supplied to us by S. Tajima. The effect of this correction on the pseudodielectric function turns out to be almost negligible, in accordance with Aspnes ${ }^{25}$.

The optical conductivity, $\sigma(\omega)$, is obtained using the relation $\epsilon(\omega)=\epsilon_{\infty}+4 \pi i \sigma(\omega) / \omega$, where $\epsilon_{\infty}$ represents the screening by interband transitions. In the copper oxide materials $\epsilon_{\infty}=4.5 \pm 0.5$. For $\omega / 2 \pi c=5,000 \mathrm{~cm}^{-1}$ an uncertainty of 0.5 of $\epsilon_{\infty}$ propagates to an error of $2^{\circ}$ of the phase of $\sigma(\omega)$. This accuracy improves for lower frequencies.

\section{Frequency dependent scattering rate}

For an isotropic Fermi liquid, the energy dependent scattering rate of the quasi-particles can be readily obtained from the optical data, using the relation $1 / \tau(\omega)=\operatorname{Re}\left\{\omega_{\mathrm{p}}^{2} / 4 \pi \sigma(\omega)\right\}$. In spite of the fact that the notion of a quasi-particle in the spirit of Landau's Fermi liquid is far from being established for the copper oxides, during the past 15 years it has become a rather common practice to represent infrared data of these materials as $1 / \tau(\omega)$. The

dynamical mass is defined as $m^{*}(\omega) / m=\operatorname{Im}\left\{\omega_{\mathrm{p}}^{2} / 4 \pi \omega \sigma(\omega)\right\}$. To obtain absolute numbers for $1 / \tau(\omega)$ and $m^{\star}(\omega) / m$ from the experimental optical conductivity, a value of the plasma frequency, $\omega_{\mathrm{p}}$, must be adopted. With our value of $\omega_{\mathrm{p}}$ the dynamical mass converges to 1 for $\omega \rightarrow \infty$. Sometimes the renormalized scattering rate, $\tau^{\star}(\omega)^{-1}=\tau(\omega)^{-1} m / m^{*}(\omega)=$ $\omega \sigma_{1}(\omega) / \sigma_{2}(\omega)$, is reported instead of $\tau(\omega)$. If the frequency dependence of the conductivity is a power law, $\sigma(\omega)=(-i \omega)^{\gamma-2}$, then $1 / \tau^{\star}(\omega)=-\omega \operatorname{cotan}(\pi \gamma / 2)$, which is a linear function of frequency ${ }^{18}$. The value of the slope reveals the exponent, and corresponds to the phase of the conductivity displayed in Figs 3 and 4.

Received 10 June; accepted 5 August 2003; doi:10.1038/nature01978.

1. Varma, C. M., Nussinov, Z. \& van Saarloos, W. Singular or non-Fermi Liquids. Phys. Rep. 361, 267-417 (2002).

2. Sachdev, S. Quantum Phase Transitions (Cambridge Univ. Press, Cambridge, 1999).

3. Saxena, S. S. et al. Superconductivity on the border of itinerant-electron ferromagnetism in $\mathrm{UGe}_{2}$. Nature 406, 587-592 (2000).

4. Tallon, J. L. \& Loram, J. W. The doping dependence of $\mathrm{T}^{*}$ - what is the real high- $T_{c}$ phase diagram? Physica C 349, 53-68 (2001).

Orenstein, J. \& Millis, A. J. Advances in the physics of high-temperature superconductivity. Science 288, 468-474 (2000).
6. Kaminski, A. et al. Spontaneous breaking of time-reversal symmetry in the pseudogap state of a high$T_{\mathrm{c}}$ superconductor. Nature 416, 610--613 (2002).

7. Mook, H. A. et al. Magnetic order in $\mathrm{YBa}_{2} \mathrm{Cu}_{3} \mathrm{O}_{6+\mathrm{x}}$ superconductors. Phys. Rev. B 66, 144513 (2002).

8. Quijada, M. A. et al. Anisotropy in the ab-plane optical properties of $\mathrm{Bi}_{2} \mathrm{Sr}_{2} \mathrm{CaCu}_{2} \mathrm{O}_{8}$ single-domain crystals. Phys. Rev. B 60, 14917-14934 (1999).

9. Puchkov, A. V., Basov, D. N. \& Timusk, T. The pseudogap state in high-Tc superconductors: An infrared study. J. Phys. 8, 10049-10082 (1996).

10. Santander-Syro, A. F. et al. Absence of a loss of in-plane infrared spectral weight in the pseudogap regime of $\mathrm{Bi}_{2} \mathrm{Sr}_{2} \mathrm{CaCu}_{2} \mathrm{O}_{8+\hat{0}}$. Phys. Rev. Lett. 88, 097005-097008 (2002).

11. Littlewood, P. B. \& Varma, C. M. Phenomenology of the normal and superconducting states of a marginal Fermi liquid. J. Appl. Phys. 69, 4979-4984 (1991).

12. Prelovsek, P. On the universal optical conductivity and single-particle relaxation in cuprates. Europhys. Lett. 53, 228-232 (2001).

13. Ioffe, L. B. \& Millis, A. J. Zone-diagonal-dominated transport in high- $T_{c}$ cuprates. Phys. Rev. B 58, 11631-11637 (1998).

14. Schlesinger, Z. et al. Superconducting energy gap and normal-state conductivity of a single-domain $\mathrm{YBa}_{2} \mathrm{Cu}_{3} \mathrm{O}_{7}$ crystal. Phys. Rev. Lett. 65, 801-804 (1990).

15. El Azrak, A. et al. Infrared properties of $\mathrm{Yba}_{2} \mathrm{Cu}_{3} \mathrm{O}_{7}$ and $\mathrm{Bi}_{2} \mathrm{Sr}_{2} \mathrm{Ca}_{\mathrm{n}-1} \mathrm{Cu}_{\mathrm{n}} \mathrm{O}_{2 \mathrm{n}+4}$ thin films. Phys. Rev. B 49, 9846-9856 (1994)

16. Molegraaf, H. J. A., Presura, C., van der Marel, D., Kes, P. H. \& Li, M. Superconductivity-induced transfer of in-plane spectral weight in $\mathrm{Bi}_{2} \mathrm{Sr}_{2} \mathrm{CaCu}_{2} \mathrm{O}_{8+\hat{\delta}}$. Science 295, 2239-2241 (2002).

17. Li, M., van der Beek, C. J., Konczykowski, M., Menovsky, A. A. \& Kes, P. H. Superconducting properties of strongly underdoped $\mathrm{Bi}_{2} \mathrm{Sr}_{2} \mathrm{CaCu}_{2} \mathrm{O}_{8+\mathrm{x}}$ single crystals. Phys. Rev. B 66, 024502 (2002).

18. Anderson, P. W. Infrared conductivity of cuprate metals: Detailed fit using Luttinger-liquid theory. Phys. Rev. B 55, 11785-11788 (1997).

19. Bernhoeft, N. An analysis of the dynamical magnetic susceptibility in non-Fermi liquids. J. Phys. Condens. Matter 13, R771-R816 (2001).

20. van der Marel, D. Anisotropy of the optical conductivity of high $T_{\mathrm{c}}$ cuprates. Phys. Rev. B 60, R765-R768 (1999).

21. Liu, Y. \& Goldman, A. M. Superconductor-insulator transitions in two dimensions. Mod. Phys. Lett. B 8, 277-309 (1994).

22. Fisher, M. P. A., Grinstein, G. \& Girvin, S. M. Presence of a quantum diffusion in two dimensions: Universal resistance at the superconductor-insulator transition. Phys. Rev. Lett. 64, 587-590 (1990).

23. Damle, K. \& Sachdev, S. Nonzero-temperature transport near quantum critical points. Phys. Rev. B 56, 8714-8733 (1997).

24. Tajima, S., Gu, D. G., Miyamoto, S., Odagawa, A. \& Koshizuka, N. Optical evidence for strong anisotropy in the normal and superconducting states in $\mathrm{Bi}_{2} \mathrm{Sr}_{2} \mathrm{CaCu}_{2} \mathrm{O}_{8+z}$. Phys. Rev. B 48, 16164-16167 (1993)

25. Aspnes, D. E. Approximate solution of ellipsometric equations for optically biaxial crystals. J. Opt. Soc. Am. 70, 1275-1277 (1980)

Acknowledgements We thank C. M. Varma, P. Prelovsek, C. Pepin, S. Sachdev and A. Tsvelik for comments during the preparation of this work, and N. Kaneko for technical assistance. This investigation was supported by the Netherlands Foundation for Fundamental Research on Matter (FOM) with financial aid from the Nederlandse Organisatie voor Wetenschappelijk Onderzoek (NWO). The crystal growth work at Stanford University was supported by the Department of Energy's Office of Basic Energy Sciences, Division of Materials Science.

Competing interests statement The authors declare that they have no competing financial interests.

Correspondence and requests for materials should be addressed to D.v.d.M.

(dirk.vandermarel@physics.unige.ch).

\section{High-performance thin-film transistors using semiconductor nanowires and nanoribbons}

\section{Xiangfeng Duan ${ }^{1,2}$, Chunming Miu ${ }^{1,2}$, Vijendra Sahi ${ }^{1,2}$, Jian Chen $^{2}$, J. Wallace Parce ${ }^{2}$, Stephen Empedocles ${ }^{2}$ \& Jay L. Goldman ${ }^{1,2}$}

${ }^{1}$ Nanosys, Inc., Advanced Research Center, 200 Boston Ave, Medford, Massachusetts 02155, USA

${ }^{2}$ Nanosys, Inc., 2625 Hanover Street, Palo Alto, California 94304, USA

Thin-film transistors (TFTs) are the fundamental building blocks for the rapidly growing field of macroelectronics ${ }^{1,2}$. The use of plastic substrates is also increasing in importance owing to their light weight, flexibility, shock resistance and low cost ${ }^{3,4}$. Current polycrystalline-Si TFT technology is difficult to implement on plastics because of the high process temperatures required ${ }^{1,2}$. Amorphous-Si and organic semiconductor ${ }^{5,6}$ TFTs, which can be 